\title{
Pulmonary Torsion as an Atypical Complication of Congenital Esophageal Atresia Repair- A Case Report and Review of Literature
}

\author{
Maria Koziarkiewicz ${ }^{1}$ Anna Taczalska ${ }^{1}$ Anna Piaseczna-Piotrowska ${ }^{1}$ \\ ${ }^{1}$ Department of Pediatric Surgery and Urology, Polish Mother's Health \\ Center Research Institute, Lodz, Poland \\ Address for correspondence Maria Koziarkiewicz, MD, PhD, \\ Department of Pediatric Surgery and Urology, Polish Mother's Health \\ Research Institute, 93-338 Lodz, Rzgowska 281/289, Poland \\ Eur J Pediatr Surg Rep 2014;2:43-45. \\ (e-mail: amaltea79@interia.pl).
}

\author{
Abstract \\ Keywords \\ - pulmonary torsion \\ - esophageal atresia \\ - postoperative \\ complication
}

Pulmonary torsion is a severe, life-threatening event, rarely occurring in children. We present a case of atypical postoperative complication of esophageal atresia repair in the form of pulmonary torsion comprising the middle lobe of the right lung. Clinical deterioration in the face of normal arterial blood gases should rise a high index of suspicion for pulmonary torsion. Early diagnosis is crucial, treatment is surgical, detorsion if possible, or if the lobe is not viable-resection.

\section{Introduction}

Esophageal atresia is an anatomical and physiological disorder of the esophagus requiring urgent surgical intervention. The most common early complications of esophageal atresia repair include leakage of the anastomosis, recurrent tracheoesophageal fistula, and anastomotic stricture. We present a case of atypical postoperative complication in the form of pulmonary torsion comprising the middle lobe of the right lung.

\section{Case Report}

A 3,260 g preterm female neonate, delivered at 38 weeks' gestation, evaluated at 7/8 points in Apgar scale, was referred to our department with prenatally diagnosed esophageal atresia. On contrast, chest X-ray performed directly after birth the presence of congenital defect-esophageal atresia with tracheoesophageal fistula-was confirmed (the upper end of esophagus visible at the level of Th4-5, dilated intestinal loops filled with air). The child was operated on the second day of life by the right laterodorsal thoracotomy. The tracheoesophageal fistula was dissected and sutured, and esophago-esophageal anastomosis was performed. Before closure, the lungs were aerated by forced ventilation, continued until all collapsed regions fully expanded. During post- operative course, on control chest X-rays, there was atelectasis in the upper lobe and the middle lobe of the right lung (-Fig. 1). On ultrasound examination, no pleural effusion was noted. On the sixth postoperative day, contrastenhanced computed tomographic (CT) scan was performed and revealed abrupt termination of the bronchial airways with atypical course of horizontal interlobal fissure (-Fig. 2). Right pulmonary artery was visible, its lobar branches hardly visible; right main bronchus showed typical diameter for approximately $7 \mathrm{~mm}$ from the bifurcation, then narrowed to approximately $1.5 \mathrm{~mm}$ (compression from outside?) and continued as a single bronchus to the lower lobe. There were neither any branches to the upper lobe nor to the middle lobe. Thus, bronchography was performed, which showed obstruction of the upper lobe bronchus and bronchus intermedius and the middle lobe bronchus of the right lung (-Fig. 3).

The patient was qualified for tracheobronchoscopy, which confirmed occlusion of the upper and middle lobar bronchi (trachea-wide, smooth, site of sutured fistula wide, patent, tracheal bifurcation normal. Main bronchus: left, normal; right, narrowing at $\sim 10 \mathrm{~mm}$ from bifurcation, with a small fissure in the sagittal plane). The neonate was qualified for second thoracotomy on the ninth day of life. On exploration, massive infarction of the upper and middle lobe of the right lung was revealed. Anastomosis site and site of
License terms

(c) 2014 Georg Thieme Verlag KG Stuttgart · New York

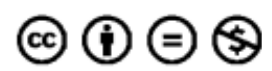

May 31, 2013

accepted after revision

October 1, 2013

published online

January 16, 2014
DOI http://dx.doi.org/

10.1055/s-0033-1361834. ISSN 2194-7619. 


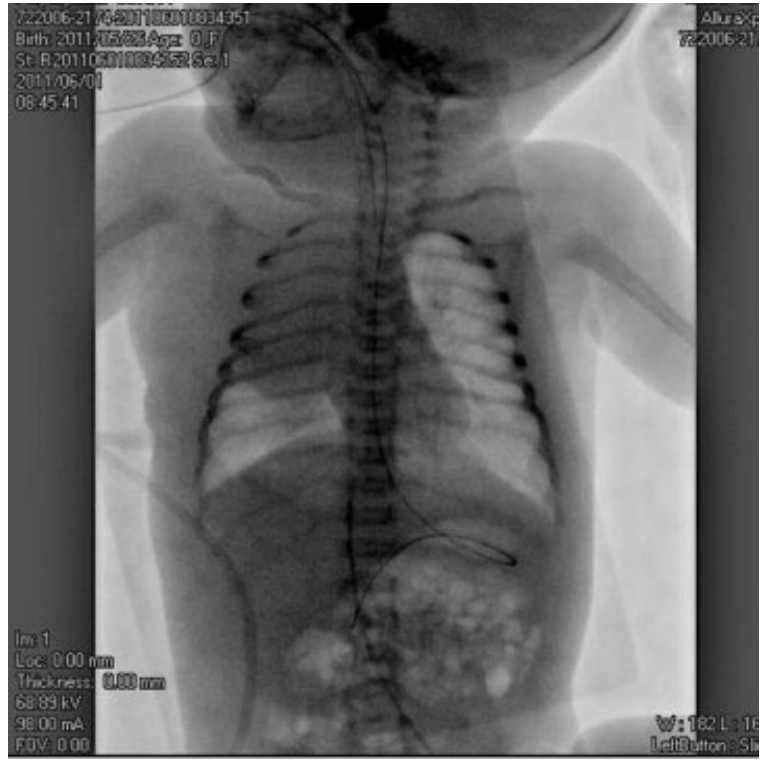

Fig. 1 Chest X-ray, fifth postoperative day.

tracheoesophageal fistula were healed. A 180-degree rotation of the right middle lobe in a clockwise direction was found, which caused traction and compression of vessels to the upper lobe. The lobe was untwisted, with return of inflation in the upper lobe under vision. In the middle lobe after detorsion inflation was seen only in fragments, however significant decrease of edema and hemostasis was seen, thus no resection was attempted.

Postoperatively, the patient recovered normal respiratory function (initially ventilation synchronized intermittent mandatory ventilation with low parameters, from 20th postoperative day spontaneous respiration, chest drain closed on 3rd postoperative day, removal of the drain on the 5th postoperative day, feeding via gastric tube from 3rd postoperative day, discharged home on 35th postoperative day). The patient was then transferred to a cardiology department for diagnostics of arrhythmia and later, at the age of 5 months, to a specialist pulmonary hospital, where the middle lobe was removed because of atelectasis.

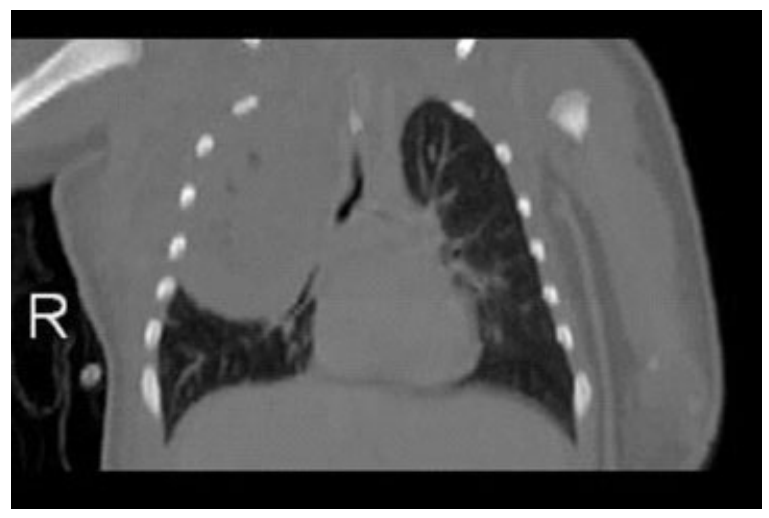

Fig. 2 Computed tomography of the chest, sixth postoperative day.

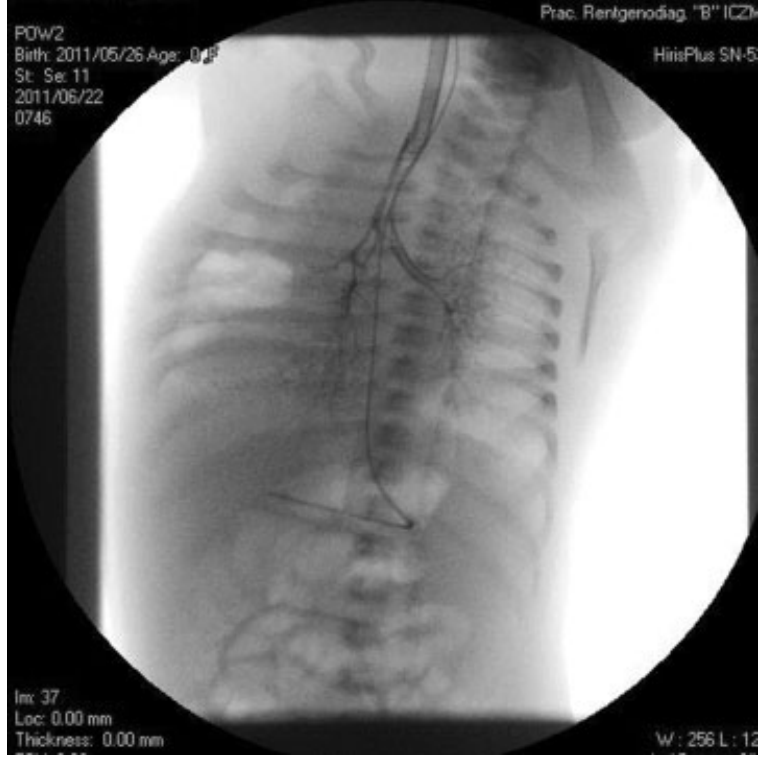

Fig. 3 Bronchography, sixth postoperative day.

\section{Discussion}

Torsion of a lobe of a lung is a severe, life-threatening event, though extremely uncommon. It may occur spontaneously after thoracic trauma, thoracic or cardiac surgery. ${ }^{1-7}$ In children, it is considerably less frequent than in adults. So far, according to our knowledge, only nine cases have been described in pediatric literature, including one case of lung torsion complicating tracheoesophageal fistula repair. ${ }^{1,2}$ Mortality in adult patients ranges from 12 to $16 \%{ }^{8}$ Pulmonary torsion causes clinical deterioration in the face of normal arterial blood gases and requires a high index of suspicion for diagnosis. The diagnosis is usually suggested on chest X-ray and confirmed by CT scans. On chest X-rays consolidation and opacification of the affected lung fragment, bronchial cutoff or distortion with mediastinum shift and lobar air trapping can be seen. ${ }^{9,10}$ The diagnosis was delayed by 14 days in some reports ${ }^{8,11}$ because of unequivocal results of imaging studies and the fact that clinical findings may be seemingly inconspicuous. Pediatric patients may present with tachycardia, fever, tachypnea, abnormal blood tests, reduced breath sounds or chest movements, dullness to percussion, and increased respiratory effort. ${ }^{5,7,8}$ If pulmonary torsion is suspected basing on clinical history and plain radiographs, chest CT scan should be performed. CT findings comprise bronchial obstruction or distortion and abnormal pattern of blood vessels. $3,4,7,9,10$ Bronchoscopy is a useful tool in the diagnosis of pulmonary torsion. ${ }^{9,10} \mathrm{~A}$ complete interlobar fissure, with no parenchymal bridges between the neighboring lobes, and an absent inferior pulmonary ligament have been described as predisposing factors. ${ }^{2}$ The accepted treatment of pulmonary torsion is detorsion or removal of the affected lobe. ${ }^{10,11}$ So far, among the eight cases described in the literature, seven cases have been operated, with five resections ${ }^{2,4,5,7,12}$ and two detorsions. ${ }^{1,6}$ Detorsion of a lung or a lobe of doubtful viability is controversial, as it may be connected with release of inflammatory mediators and necrotic tissue, leading to a systemic 
response and multiorgan failure. ${ }^{1,13,14}$ In the two described cases of detorsion, one patient recovered completely, ${ }^{1}$ whereas in the second case the lung remained shrunken and nonfunctioning. ${ }^{6}$ In this case, detorsion allowed for better assessment of the lobe viability, and decrease in edema and hemostasis were observed. In the case of our patient, the torsion was probably because of an anatomic anomaly-absence of parenchymal bridges between contiguous lobes. Earlier reoperation would have probably been beneficial, but as this complication is extremely rare in the pediatric population, diagnostic process may take longer. Persistent atelectasis of a lung/lobe after thoracotomy should call attention to a possibility of torsion.

\section{Conclusions}

The following can be concluded from this case report:

1. Pulmonary torsion is a severe, life-threatening event, rarely occurring in children.

2. Early diagnosis (X-ray/CT/bronchography/bronchoscopy) may be lifesaving. Clinical deterioration in the face of normal arterial blood gases should rise a high index of suspicion for diagnosis.

3. Treatment is surgical, detorsion if possible, or if the lobe is not viable-resection.

\section{References}

1 Oliveira C, Zamakhshary M, Abdallah MR, et al. Lung torsion after tracheoesophageal fistula repair: a case report and review of literature. J Pediatr Surg 2007;42(11):E5-E9
2 Alberti D, Borsellino A, Migliazza L, et al. Pulmonary torsion after cardiac surgery in two infants: review of pediatric literature. J Pediatr Surg 2004;39(11):1719-1723

3 Stratemeier EH, Barry JW. Torsion of the lung following thoracic trauma; a case report. Radiology 1954;62(5):726-727

4 Parks RE. Traumatic torsion of the lung. Radiology 1956;67(4): 582-583

5 Selmonosky CA, Flege JB Jr, Ehrenhaft JL. Torsion of a lobe of the lung due to blunt thoracic trauma. Ann Thorac Surg 1967;4(2): 166-170

$6 \mathrm{Fu} \mathrm{JJ}$, Chen CL, Wu JY. Lung torsion: survival of a patient whose hemorrhagic infarcted lung remained in situ after detorsion. J Thorac Cardiovasc Surg 1990;99(6):1112-1114

7 Daughtry DC. Traumatic torsion of the lung. N Engl J Med 1957; 256(9):385-388

8 Cable DG, Deschamps C, Allen MS, et al. Lobar torsion after pulmonary resection: presentation and outcome. J Thorac Cardiovasc Surg 2001;122(6):1091-1093

9 Felson B. Lung torsion: radiographic findings in nine cases. Radiology 1987;162(3):631-638

10 Banki F, Velmahos GC. Partial pulmonary torsion after thoracotomy without pulmonary resection. J Trauma 2005;59(2): 478-481

11 Parambil JG, Savci CD, Tazelaar HD, Ryu JH. Causes and presenting features of pulmonary infarctions in 43 cases identified by surgical lung biopsy. Chest 2005;127(4):1178-1183

12 Hislop A, Reid L. New pathological findings in emphysema of childhood. 2. Overinflation of a normal lobe. Thorax 1971;26(2): 190-194

13 Grazia TJ, Hodges TN, Cleveland JC Jr, Sheridan BC, Zamora MR. Lobar torsion complicating bilateral lung transplantation. J Heart Lung Transplant 2003;22(1):102-106

14 Yang EM, Song ES, In Jang H, Jeong IS, Choi YY. Lung torsion after tracheoesophageal fistula repair in an infant. Korean J Pediatr 2013;56(4):186-190 